\title{
Towards automated detection of depression from brain structural magnetic resonance images
}

\author{
Kuryati Kipli • Abbas Z. Kouzani • Lana J. Williams
}

Received: 26 June 2012 / Accepted: 7 January 2013 /Published online: 22 January 2013

(C) Springer-Verlag Berlin Heidelberg 2013

\begin{abstract}
Introduction Depression is a major issue worldwide and is seen as a significant health problem. Stigma and patient denial, clinical experience, time limitations, and reliability of psychometrics are barriers to the clinical diagnoses of depression. Thus, the establishment of an automated system that could detect such abnormalities would assist medical experts in their decision-making process. This paper reviews existing methods for the automated detection of depression from brain structural magnetic resonance images (sMRI). Methods Relevant sources were identified from various databases and online sites using a combination of keywords and terms including depression, major depressive disorder, detection, classification, and MRI databases. Reference lists of chosen articles were further reviewed for associated publications.

Results The paper introduces a generic structure for representing and describing the methods developed for the detection of depression from sMRI of the brain. It consists of a number of components including acquisition and preprocessing, feature extraction, feature selection, and classification.

Conclusion Automated sMRI-based detection methods have the potential to provide an objective measure of depression, hence improving the confidence level in the diagnosis and prognosis of depression.
\end{abstract}

K. Kipli $(\bowtie) \cdot$ A. Z. Kouzani

School of Engineering, Deakin University, Waurn Ponds, Victoria 3216, Australia

e-mail: kkipli@deakin.edu.au

K. Kipli

Department of Electronic, Faculty of Engineering, Universiti

Malaysia Sarawak, 94300, Kota Samarahan, Sarawak, Malaysia

L. J. Williams

School of Medicine, Deakin University, Waurn Ponds, Victoria

3216, Australia
Keywords Depression · Magnetic resonance imaging · Pattern recognition $\cdot$ Image processing $\cdot$ Classification

\section{Introduction}

Depression is the most common mental disorder worldwide and currently the fourth largest contributor to the burden of disease as reported by the World Health Organization [1]. It is estimated that, by 2020 , depression will remain a leading cause of disability, second only to cardiovascular disease [1]. Depression is a complex phenomenon with many subtypes and probably more than one etiology. It is a long-term recurrent disease in most people, with significant morbidity, mortality, and psychosocial impairment. Depression is associated with widely varying psychological and physiological features, and this heterogeneity is acknowledged within classification systems [2].

A recent epidemiological study by Bromet et al. [3] indicated that approximately 121 million people worldwide have been affected by depression. Suicide is the worst consequence of depression, with up to 850,000 deaths reported every year [4]. Suicide rates have been shown to increase with age for both males and females: from 1 death per 100,000 young people aged 12 to 14 years to 5 deaths per 100,000 people aged 15 to 17 years and 13 deaths per 100,000 people aged 18 to 24 years [3]. Despite the efforts devoted to the recognition and treatment of depression, new data suggest that the prevalence of depression may be on the rise, in particular in younger people [5].

The aim of this paper is to review the literature on the detection of depression from structural magnetic resonance images (sMRI) and describe the current methods available in automating the detection process. More specifically, the paper endeavors to (1) propose a generic structure for automated sMRI-based depression detection systems consisting 